\title{
On the association of the oxidised LDL receptor 1 (OLR1) gene in patients with acute myocardial infarction or coronary artery disease
}

\author{
Elisabetta Trabetti ${ }^{*},{ }^{1}$, Michele Biscuola ${ }^{1}$, Ugo Cavallari $^{1}$, Giovanni Malerba ${ }^{1}$, \\ Domenico Girelli ${ }^{2}$, Oliviero Olivieri ${ }^{2}$, Nicola Martinelli ${ }^{2}$, Roberto Corrocher ${ }^{2}$ \\ and Pier Franco Pignatti ${ }^{1}$
}

\footnotetext{
${ }^{1}$ Department of Mother and Child and of Biology-Genetics, Section of Biology and Genetics, University of Verona, Verona, Italy; ${ }^{2}$ Department of Clinical and Experimental Medicine, University of Verona, Verona, Italy
}

The human oxidised low-density lipoprotein receptor 1 (OLR1) gene is a functional candidate for atherosclerosis. An association of the OLR1 gene with acute myocardial infarction (AMI) or coronary artery disease (CAD) has recently been reported. In the present study a total of 677 Italian subjects, 327 CAD-free, 350 CAD, of which 190 with AMI and 160 AMI-free, was genotyped for the following four OLR1 single nucleotide polymorphisms: exon 4 K167N, IVS4-73C > T, IVS4-14A $>$ G, and 3'UTR 188 C $>$ T. No statistically significant difference was observed in allele or genotype distribution of the exon 4, intron 4, or $3^{\prime}$ UTR SNPs in CAD patients compared to CAD-free subjects, or within CAD, in AMI patients compared to AMI-free patients. A correlation was found between the $\mathrm{K} 167 \mathrm{~N} \mathrm{G} / \mathrm{G}$ genotype and the increased number of obstructed vessels. Even if the OLR1 genotype frequency distribution data in CAD or AMI subjects here reported do not fully confirm the positive results of some other association studies, an association with a marker of CAD severity was observed.

European Journal of Human Genetics (2006) 14, 127-130. doi:10.1038/sj.ejhg.5201513; published online 26 October 2005

Keywords: SNP; association study; coronary artery disease; acute myocardial infarction; CVD

\section{Introduction}

Several studies have attempted to identify candidate genes that may be associated with acute myocardial infarction (AMI) or coronary artery disease (CAD). ${ }^{1,2}$ One such gene is the oxidized low-density lipoprotein (LDL) receptor 1 (OLR1, or lectin-like oxidised LDL receptor 1, LOX-1) gene. OLR1 is induced by proatherogenic stimuli and by inflammatory cytokines, ${ }^{3}$ and it is upregulated in ischaemia reperfusion injury in the rat. ${ }^{4,5}$ An atherosclerosis susceptibility locus in mice has been identified and

*Correspondence: Dr E Trabetti, Department of Mother and Child and of Biology-Genetics, Section of Biology and Genetics, University of Verona, Strada Le Grazie 8, 37134 Verona, Italy.

Tel: + 3945 8027209; Fax: + 3945 8027180;

E-mail: elisabetta.trabetti@univr.it

Received 14 September 2004; revised 16 August 2005; accepted 16 September 2005; published online 26 October 2005 mapped in a syntenic region with human chromosome 12 p13-p12, which contains the OLR1 gene. ${ }^{6}$

The association of the OLR1 K167N polymorphism, and of other OLR1 gene SNPs in linkage disequilibrium (LD), with AMI has been reported. ${ }^{7,8}$ These polymorphisms have also been associated with CAD. ${ }^{9,10}$

The aim of the present study was to confirm the association of the OLR1 gene with AMI or CAD in a novel, well phenotyped, and homogenous, population.

Materials and methods

Subjects

Subjects with angiographic documentation of their coronary artery obstruction $(N=677)$ were enrolled in this study. Of these subjects, 350 subjects had angiographically documented severe coronary atherosclerosis (CAD group), 
that is, lesions with greater than 50\% luminal stenosis, and 327 had normal coronary arteries (CAD-free group). Among the CAD subjects, the AMI $(N=190)$, and the AMI-free $(N=160)$ subgroups were selected. A complete clinical history that included also the assessment of conventional cardiovascular risk factors was collected for all participants. After a multivariate analysis, as expected, CAD patients had more conventional risk factors as compared with CAD-free controls: higher BMI, total and LDL cholesterol, a higher prevalence of hypertension and smoking, and lower HDL cholesterol $(P \leq 0.0005)$. Details on the enrollment criteria have been described elsewhere. ${ }^{11,12}$

All individuals gave their consent before entering the study, which was approved by the Ethical Committee of the Verona Hospital.

\section{SNP genotyping}

We genotyped all the 677 subjects for the following three SNPs of the OLR1 gene: K167N (501G >C) in exon 4, IVS4$73 \mathrm{C}>\mathrm{T}$, and IVS4-14A $>$ G. Moreover, we genotyped 100 subjects for the $3^{\prime} \mathrm{UTR} 188 \mathrm{C}>\mathrm{T}$, in order to confirm the previously described LD block. ${ }^{8}$

For K167N and 3'UTR 188C > T detection, a new method was used with modified forward primers in order to establish a NlaIV and a RsaI restriction site, respectively (New England Biolabs, Beverley, MA, USA). The primer pairs 167F: 5'-GGCTCATTTAACTGGGAAAG-3', 167R: 5'CCGTCCAAGGTCATACACAA-3', and 188F: ${ }^{\prime}$-TGTCAACA TTTTTGATTCTAGGTA, 188R: 5'-GTTCTCCATGTTCTGTC TTTCA-3' were used, each modified nucleotide is underlined. The K167N PCR product was $239 \mathrm{bp}$. After NlaIV restriction two fragments were obtained: 217 and $22 \mathrm{bp}$ for the K allele, and a single fragment of $239 \mathrm{bp}$ for the $\mathrm{N}$ allele. The $3^{\prime}$ UTR $188 \mathrm{C}>\mathrm{T}$ PCR product was $207 \mathrm{bp}$. After RsaI restriction two fragments were obtained: 184 and $23 \mathrm{bp}$ for the $\mathrm{C}$ allele, and a single fragment of $207 \mathrm{bp}$ for the $\mathrm{T}$ allele.

For the IVS4-73C $>\mathrm{T}$ and $-14 \mathrm{~A}>\mathrm{G}$ SNPs, a single PCR was performed using the primers $\mathrm{F}$ : $5^{\prime}$-CAGTCAAGGGGA TGTCAAAGA-3' and R: 5'-GAGGCATCAAAAAGAATG GG-3', as previously described. ${ }^{8}$ The PCR product was 267 bp. After BamHI (New England Biolabs) restriction two fragments were obtained: 220 and $47 \mathrm{bp}$ for the $-73 \mathrm{C}$ allele, and a single fragment of $267 \mathrm{bp}$ for the $-73 \mathrm{~T}$ allele. After MaeIII (New England Biolabs) restriction two fragments were obtained: 157 and $110 \mathrm{bp}$ for the $-14 \mathrm{G}$ allele, and a single fragment of $267 \mathrm{bp}$ for the $-14 \mathrm{~A}$ allele.

\section{Statistical analysis}

Genotype and allele frequencies of the four groups of patients were compared by $\chi^{2}$ analysis. Hardy-Weinberg equilibrium was tested. All the calculations were performed with SPSS 11.5 statistical package (SPSS Inc., Chicago, IL, USA).

\section{Results}

Study subjects were genotyped as described for the following OLR1 gene SNPs: K167N (501G>C), IVS473C $>$ T, IVS4-14A > G, $3^{\prime}$ UTR $188 \mathrm{C}>\mathrm{T}$.

Table 1 reports the allele and genotype frequencies observed for $\mathrm{CAD}$ and $\mathrm{AMI}$ patients. Data are given in Table 1 only for K167N and IVS4-73C > T SNPs, as IVS4$73 \mathrm{C}>\mathrm{T}$ is in complete LD with both IVS4-14A $>\mathrm{G}$ and $3^{\prime} \mathrm{UTR} 188 \mathrm{C}>\mathrm{T}$ (C-A-C, respectively). The distribution of genotypes within each group was in Hardy-Weinberg equilibrium. The K167N polymorphism has an observed LD with IVS4-73C $>$ T: $D^{\prime}=0.87$. K167N (501G >C) - IVS4$73 \mathrm{C}>\mathrm{T}$ haplotype frequencies were estimated, and the following three haplotypes with frequency $>0.01$ were observed: G-T (53\%), G-C (39\%), C-C (7.5\%), C-T (0.5\%).

No statistically significant difference was observed in allele or genotype frequencies for each polymorphism in CAD patients compared to CAD-free controls, in AMI patients compared to AMI-free patients, or in AMI patients compared to CAD-free controls.

An association analysis was performed by grouping OLR1 genotypes as follows: $\mathrm{K} 167 \mathrm{~N}(501 \mathrm{G}>\mathrm{C}) \mathrm{GG} v s \mathrm{GC}+\mathrm{CC},{ }^{7}$ and IVS $4-73 \mathrm{C}>\mathrm{T}$ CC $v s$ CT or TT. ${ }^{8}$ The results are given in Table 2. No significant association was observed.

No association was detected for OLR1 SNPs genotypes when the conventional cardiovascular risk factors were included in the model.

No association of $\mathrm{K} 167 \mathrm{~N}$ and $-73 \mathrm{C}>\mathrm{T}$ SNPs with CAD was observed in women ${ }^{9}$ (50 CAD vs 108 CAD-free; $P=0.17, P=0.38$, respectively).

The distribution of K167N or IVS4-73C > T genotypes in the CAD patients did not show any significant difference or trend according to the number of stenosed vessels (one, 41 patients; two, 69 patients; or three, 231 patients; for nine subjects data were not available; $P=0.35, P=0.41$, respectively, for the two polymorphisms) (data not shown).

Table 1 OLR1 K167N (501G >C) (a), and IVS4-73C > T (b) genotype and allele frequencies, respectively, in the studied subjects

\begin{tabular}{lllll}
\hline & $\begin{array}{c}\text { CAD } \\
(N=350)\end{array}$ & $\begin{array}{c}\text { CAD-free } \\
(N=327)\end{array}$ & $\begin{array}{c}\text { AMI } \\
(N=190)\end{array}$ & $\begin{array}{c}\text { AMI-free } \\
(N=160)\end{array}$ \\
\hline (a) & & & & \\
G/G & 0.851 & 0.850 & 0.847 & 0.869 \\
G/C & 0.143 & 0.147 & 0.147 & 0.125 \\
C/C & 0.006 & 0.003 & 0.006 & 0.006 \\
G & 0.92 & 0.92 & 0.92 & 0.93 \\
C & 0.08 & 0.08 & 0.08 & 0.07 \\
& & & & \\
(b) & & & & \\
C/C & 0.197 & 0.260 & 0.195 & 0.200 \\
C/T & 0.506 & 0.453 & 0.531 & 0.475 \\
T/T & 0.297 & 0.287 & 0.274 & 0.325 \\
C & 0.45 & 0.49 & 0.46 & 0.44 \\
T & 0.55 & 0.51 & 0.54 & 0.56 \\
\hline
\end{tabular}


Table 2 Association analysis for OLR1 K167N or IVS4-73C > T SNPs in CAD or AMI subjects

\begin{tabular}{|c|c|c|c|c|c|c|c|c|}
\hline & \multicolumn{4}{|c|}{$C A D$ vs $C A D$-free } & \multicolumn{4}{|c|}{ AMI vs AMI-free } \\
\hline & $O R$ & $S E$ & $\mathrm{Cl}(95 \%)$ & P-value & OR & SE & $\mathrm{Cl}(95 \%)$ & P-value \\
\hline K167N GG vs GC+CC & 1.02 & 1.005 & $0.651-1.605$ & 0.98 & 0.965 & 1.32 & $0.55-1.68$ & 0.88 \\
\hline IVS4-73C > T CT+TT vs CC & 1.42 & 1.20 & $0.99-2.04$ & 0.065 & 1.31 & 1.27 & $0.82-2.10$ & 0.29 \\
\hline
\end{tabular}

Table 3 Association results between OLR1 gene polymorphisms and acute myocardial infarction (AMI) or coronary artery disease (CAD) severity in the previous studies and the present study

\begin{tabular}{|c|c|c|c|c|c|}
\hline & Sample size & Risk genotype & OR $(C I)$ & P-value & Associated phenotype \\
\hline Tatsuguchi et $a l^{7}$ & 204 & $501(\mathrm{G} / \mathrm{C}+\mathrm{C} / \mathrm{C})$ & $2.89(1.51-5.53)$ & $<0.002$ & AMI \\
\hline Mango et $a l^{8}$ & 253 & $3^{\prime}$ UTR $188 \mathrm{CT}$ or TT & $3.74(1.73-8.18)$ & $<0.0001$ & AMI \\
\hline Chen et $a P^{\rho}$ & 563 & 3'UTR 188 (CT+TT) & $0.61(0.41-0.91)$ & 0.014 & CAD severity \\
\hline Ohmori et $a l^{10}$ & 586 & $501(\mathrm{G} / \mathrm{C}+\mathrm{C} / \mathrm{C})$ & $0.61(0.41-0.92)$ & $<0.025$ & CAD severity \\
\hline Present study & 350 & $501 \mathrm{G} / \mathrm{G}$ & $0.47(0.19-1.01)$ & 0.045 & CAD severity \\
\hline
\end{tabular}

A significant association was observed when comparing the K167N GG genotype frequency in patients having three obstructed vessels $v s$ patients having one or two (OR: 0.469, CI: $0.199-1.01, P=0.045)$.

\section{Discussion}

In the present study, the exon $4 \mathrm{~K} 167 \mathrm{~N}$, the IVS4-73C $>\mathrm{T}$ and $-14 \mathrm{~A}>\mathrm{G}$, and the $3^{\prime} \mathrm{UTR} 188 \mathrm{C}>\mathrm{T}$ polymorphisms of the OLR1 gene, described to be associated with $\mathrm{AMI}^{7,8}$ or CAD,${ }^{9-10}$ were analysed. Table 3 shows a summary of the results from the above reported association studies and the present study for comparative purposes. Three common haplotypes account for $85 \%$ of the observed OLR1 haplotypes in the CEPH individuals from the HapMap Project (10 SNPs). Three LD blocks were described. ${ }^{13}$ The SNPs reported in this study, not genotyped by the HapMap project, map in the third LD block. Mango et $\mathrm{al}^{8}$ screened all exons and intron/exon boundaries of the gene, finding seven SNPs, and indicated that AMI risk was associated with SNPs mapping from exon 4 to the $3^{\prime}$ UTR (third LD block). As the sample set described in the present manuscript and in Mango et $a l^{8}$ are both from the Italian population, it is unlikely that other risk susceptibility SNPs in the first two blocks of the OLR1 gene could be present.

We did not confirm the association of the OLR1 K167N polymorphism and AMI previously described in the Japanese population $(\mathrm{GC}+\mathrm{CC}$ : $\mathrm{OR}=2.89$; $\mathrm{CI}: 1.51-5.53){ }^{7}$ We did not confirm the different frequency distribution of the OLR1 IVS4-73T/T homozygotes between AMI and AMI-free groups previously described in another Italian population sample: $3^{\prime} \mathrm{UTR} 188 \mathrm{C}>\mathrm{T}$ (which is in complete LD with IVS4-73C $>$ T) and AMI $(\mathrm{OR}=3.74$; CI: $1.73-$ 8.18). ${ }^{8}$ These discrepancies could be due to an ascertainment difference, or to a population difference, or to the limited study numerosity (350 in the present study, 204 in the Japanese study, and 253 in the previous Italian study, respectively).

Two studies reported a $3^{\prime} \mathrm{UTR}$ or a $\mathrm{K} 167 \mathrm{~N}$ association with CAD severity in non-Hispanic white women in the US population $(\mathrm{CT}+\mathrm{TT} \text { : } \mathrm{OR}=0.61 ; \mathrm{CI}: 0.41-0.91)^{9}$ and in the Japanese population $(\mathrm{CC}+\mathrm{CG}$ : $\mathrm{OR}=0.61$; $\mathrm{CI}: 0.41-$ $0.92),{ }^{10}$ respectively. All CAD patients here described had severe coronary atherosclerosis, that is, lesions with greater than 50\% luminal stenosis. The distribution of genotypes in CAD patients in the present study did not show any significant difference with increasing number of stenosed coronary arteries, while an association was detected in the most severely affected patient class. In this study, the K167N GG genotype has the opposite effect on CAD severity with respect to what described in the Japanese population. $^{10}$

We performed an association analysis under the model used in two previous studies. ${ }^{7,8}$ The association of IVS4$73 \mathrm{C}>\mathrm{T}$ with $\mathrm{CAD}$ was close to statistical significance (results given in Table 2).

The present results suggest that the effect of the gene, if associated with CAD and/or AMI, might be smaller than previously reported, rejecting association at an $\mathrm{OR} \geq 1.5$.

Further studies in a larger set of individuals may help in detecting SNPs associated with a more modest risk. Our calculations indicate that the sample size should be at least 2400 or 2730 for IVS4-73C > T or 4650 or 4950 for K167N to detect a significant association in CAD or AMI, respectively (significance level $=0.001$, power $=80 \%$, OR $=1.4$ ).

In conclusion, in accordance with some previous evidence of the oxidised LDL receptor involvement in atherogenesis, the OLR1 genotype frequency distribution data in CAD or AMI subjects here reported provide some indication of a possible association. 


\section{Acknowledgements}

This project was supported by the Italian Ministry of Education, University and Research and Foundation 'Cassa di Risparmio di Verona, Vicenza, Belluno e Ancona'. We thank professor Giuseppe Novelli (University of Rome Tor Vergata) for his suggestion on the intron 4 SNP genotyping method.

\section{References}

1 Winkelmann BR, Hager J: Genetic variation in coronary heart disease and myocardial infarction: methodological overview and clinical evidence. Pharmacogenomics 2000; 1: 73-94.

2 Auer J, Weber T, Berent R, Lassnig E, Lamm G, Eber B: Genetic polymorphisms in cytokine and adhesion molecule genes in coronary artery disease. Am J Pharmacogenomics 2003; 3: 317-328.

3 Mehta JL, Li DY: Identification, regulation and function of a novel lectin-like oxidized low-density lipoprotein receptor. J Am Coll Cardiol 2002; 39: 1429-1435.

4 Li D, Williams V, Liu L et al: LOX-1 inhibition in myocardial ischemia-reperfusion injury: modulation of MMP-1 and inflammation. Am J Physiol Heart Circ Physiol 2002; 283: H1795-H1801.

$5 \mathrm{Li}$ D, Williams V, Liu L et al: Expression of lectin-like oxidized lowdensity lipoprotein receptors during ischemia-reperfusion and its role in determination of apoptosis and left ventricular dysfunction. J Am Coll Cardiol 2003; 19: 1048-1055.

6 Welch CL, Bretschger S, Latib $\mathrm{N}$ et al: Localization of atherosclerosis susceptibility loci to chromosomes 4 and 6 using the
Ldlr knockout mouse model. Proc Natl Acad Sci USA 2001; 98: 7946-7951.

7 Tatsuguchi M, Furutani M, Hinagata J et al: Oxidized LDL receptor gene (OLR1) is associated with the risk of myocardial infarction. Biochem Biophys Res Commun 2003; 303: 247-250.

8 Mango R, Clementi F, Borgiani $\mathrm{P}$ et al: Association of single nucleotide polymorphisms in the oxidised LDL receptor 1 (OLR1) gene in patients with acute myocardial infarction. J Med Genet 2003; 40: 933-936.

9 Chen Q, Reis SE, Kammerer C et al: Genetic variation in Lectinlike Oxidized Low-Density Lipoprotein Receptor 1 (LOX1) gene and the risk of coronary artery disease. Circulation 2003; 107: 3146-3151.

10 Ohmori R, Momiyama $\mathrm{Y}$, Nagano $\mathrm{M}$ et al: An oxidized lowdensity lipoprotein receptor gene variant is inversely associated with the severity of coronary artery disease. Clin Cardiol 2004; 27: 641-644.

11 Girelli D, Friso S, Trabetti E et al: Methylentetrahydrofolate Reductase C677T mutation, plasma homocysteine and folate, in subjects from Northern Italy with or without angiographically documented severe coronary atherosclerotic disease. Evidence for an important genetic-environmental interaction. Blood 1998; 91: $4158-4163$.

12 Martinelli N, Girelli D, Olivieri $\mathrm{O}$ et al: Interaction between smoking and PON2 SER311CYS polymorphism as determinant of the risk of myocardial infarction. Eur J Clin Invest 2004; 34: $14-20$.

13 The International HapMap Consortium: The International HapMap Project. Nature 2003; 426: 789-796. 